\title{
Evaluation of Creep Damage in Some Major Components of Power Generator
}

\author{
Jiří MATOUŠEK, 1) Milan ŠVARC, 1) Jaroslav VALENTA, 1) Karel LÖBL2) and Vladimír BíNA3)
}

1) National Research Institute for Machine Design, Prague Běchovice (190 11), Czechoslovakia. Physical Engineering, Technical University of Prague, Lazarska 7 (113 48), Prague 1, Czechoslovakia.

2) Faculty of Nuclear Science and of Materials, Opletalova 25 (113 12), Prague 1, Czechoslovakia.

3) National Research Institute

(Received on October 9, 1989; accepted in the final form on March 9, 1990)

\begin{abstract}
Life extension of installed machinery and equipment has become a matter of strategic importance with significant economic implications especially in power plants.

The paper presents a complex of methods aimed at assessment of the residual service lives of major components of power plant machinery (steam turbines, steam line systems) under creep conditions. The methods are based on a mathematical model of properties of creep-resistant steels based on the dislocation mechanism for which stochastic behaviour of material in the course of the strain mechanism has been assumed. A method is proposed to evaluate the equivalent service load in actual service conditions by means of a computerized data acquisition. The method is applied to monitor creep damage of steam turbine pipelines. Creep damage of turbine rotors and casings and plastic deformation in their critical points were estimated numerically.

The actual time span of reliable operation of a component is assessed by these method and compared with results obtained by diagnostic methods prepared in cooperation with manufactures of power generating machinery.
\end{abstract}

KEY WORDS: creep damage; stochastic behaviour; residual service-life; plastic elongations; limit deformation; equivalent service loading.

\section{Introduction}

The area of problems related to prolongation of reliable operation of installed machinery and equipment has become a matter of strategic importance with significant economic implications. For example in the field of power generating plants, the means required for full exploitation of the life-times available have been reported (Ref. 1)) to represent approximately only from 25 to $33 \%$ of the expenditure necessary for setting up a new installation.

These objectives, however, require extensive experimental and theoretical research of materials behaviour under extreme conditions. Structural degradation of material must be assessed with regard to gradual amplification of the effects of operational states. For example on the basis of data processed by the "Reliability database system of Czechoslovak power generating installations" (Ref. 2)) the trend of emergency outages of Skoda steam turbines has the typical bath-tub shape (Fig. 1). The first region of this well-known curve is that in which design and material flaws are predominant, this area in the given case ending by about $4 \times 10^{4}$ service hours. Stable operation is typical of the second region, with emergency outage (given as per cents of service hours) equal to $0.2 \%$ in the case of steam turbines of the type studied. The third region is the period in which increasing emergency outages can be expected owing to exhaustion of the computational or real life-times of major components of the installation (rotors, casings, valve bodies, etc.).
Important role is played here not only by the ordinary fundamental service effects but primarily by emergency situations, such as fast temperature changes etc. Hence a number of top-ranking steam turbine manufacturers from the USA, Japan, France, Germany, and Switzerland, but also from Czechoslovakia, devote significant efforts to monitoring the actual process of service exhaustion of the available time span of reliable operation and to diagnostics of the major components of steam turbines.

Efforts have also been made to improve materials of components in order to facilitate operation at higher pressures and temperatures, and the application of computer-aided techniques (CAD, CAM) has served for the same purpose as well (Ref. 2)). In the following, some of the results will be presented obtained in Czechoslovakia in the field of residual service-life

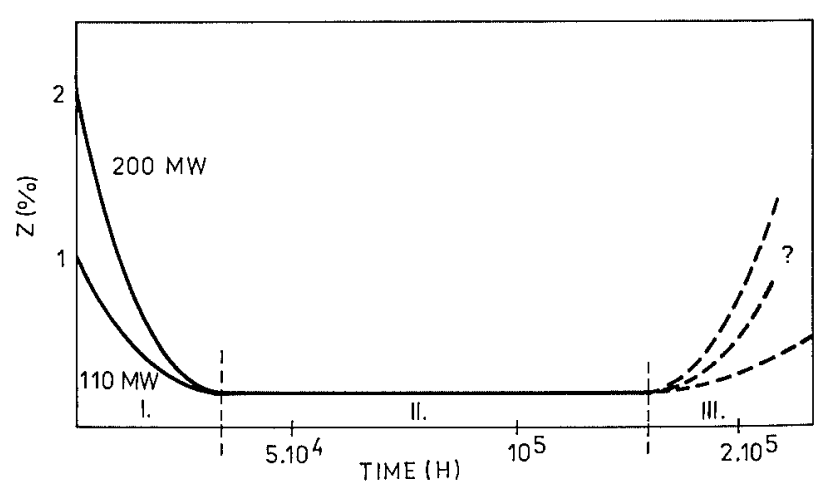

Fig. 1. The trend with time of the emergency outage coefficient of Skoda steam turbines 
assessment of major component of power-generating machinery (steam turbines, steam line systems) under creep conditions.

\section{A Mathematical Model of Properties of Heat-resistant Steels}

The process of establishment of a strain dependence model has been based upon the principle of dislocation mechanism for which stochastic behaviour of material during the strain mechanism has been assumed. Creep deformation tests (i.e., ones by which plastic elongation $\varepsilon_{c}$ is measured) provide the fundamental experimental data. The constitutive equation describing the development of deformation can be derived for the mean value of $\varepsilon_{c}$ as (Refs. 3) and 4))

$$
\varepsilon_{c}=\varepsilon_{0}\left(\frac{\varepsilon_{l}}{\varepsilon_{0}}\right)^{g(D)}
$$

where the damage parameter $D$ replaces the parameter of time, $t$.

Relationship

$$
\varepsilon_{0}=\frac{\sigma}{E_{T}}
$$

is valid for the initial deformation $\varepsilon_{0}$ in the region of elastic stress/strain relationship in which $E_{T}$ stands for the modulus of elasticity at temperature $T$.

The deformation limit $\varepsilon_{l}$ can be determined by extrapolation of the non-accelerating creep curve up to fracture. Thus only the collapse phase of the creep process is excluded from the present mathematical model, this stage, however, representing only approximately $1 \%$ of the overall time to fracture. The relationship

$$
\varepsilon_{l}=\exp \left[A_{1}+A_{2} \tanh \left(\frac{\ln t_{r}-A_{3}-A_{4} T}{A_{5}}\right)\right]+\varepsilon_{0}
$$

holds for $\varepsilon_{l}$. The time to rupture $t_{r}$ for forged steels can be expressed by the equation

$$
\begin{aligned}
\log t_{r}= & B_{1}+B_{2} \log \left[\frac{1}{T}-\frac{1}{B_{5}}\right] \\
& +B_{3} \log \left[\frac{1}{T}-\frac{1}{B_{5}}\right] \log \left[\sinh \left(B_{6} \sigma T\right)\right] \\
& +B_{4} \log \left[\sinh \left(B_{6} \sigma T\right)\right] \quad \ldots \ldots \ldots \ldots \ldots \ldots \ldots \ldots \ldots \ldots
\end{aligned}
$$

The hardening function $g(D)$ can be expressed as

$$
g(D)=[D(t)]^{N} \frac{\left\{1+\exp \left[-2(D(t))^{K}\right]\right\}^{M}}{\{1+\exp (-2)\}^{M}}
$$

where $D(t)=t / t_{r}$ is the material damage at time $t$. Numerical values of the regression parameters $A_{i}, B_{i}$, $K, M, \mathcal{N}$, have been obtained for $\mathrm{Cr} 1.25 \mathrm{Mo0.65V} 0.25$, Cr1.0Ni0.7Mo0.9V0.3, and Gr1.25Mo0.65V0.75W0.55 grade steels (steam turbine rotors) and also for Cr1.25Mo1.0V0.25 steel (the internal high-pressure casing of the $200 \mathrm{MW}$ turbine) by experimental identification of the creep process.

\section{A Method of Evaluation of the Equivalent Service Load Parameter for a Computerized Operational Data Acquisition System}

\subsection{The Equivalent Service Load Parameter as Defined from the Empirical Identification of Creep Properties of Materials}

Estimation of the equivalent service loading based on the operational history of a specific subsystem of a power-generating plant represents the fundamental prerequisite for application of any analytical procedure of life-time appraisal (Ref. 4)).

The equivalent service loading parameter of a component can be defined using empirical equations of the type (4) identifying materials creep properties. For the present purpose, the simplest regression

$$
\mu_{\imath}=a+b \frac{1}{T_{j}}+c \log \sigma_{i} \frac{1}{T_{j}}
$$

was experimentally verified to hold for steels used in Czechoslovakia in the given application.

In Eq. (6), $\mu_{t}=E\left(\log t_{r}\right)$ represents the mean value of the logarithm of the time to rupture at loading parameters $\left(\sigma_{i}, T_{j}\right)$, and $a, b$, and $c$ are the individual parameters of regression. The numerical values of these follow from material tests.

Solving Eq. (6) for temperature and assuming linear cumulation of damage, the sought parameter of equivalent service loading can be defined from Eq. (6) as a Gaussian variable of expected value given by relation

$$
E\left[T_{e j}\right]=\frac{b+c \log E\left[\sigma_{i}\right]}{\log \left[1 /\left(\sum_{(i)} \xi_{i}\right)\right]-a}
$$

in which $\xi_{i}$ represents the relative cumulative time of operation of the system at loading represented by parameters $\left(\sigma_{i}, T_{i}\right)$. Hence

$$
\xi_{i}=\frac{x_{i}}{t_{r}\left(\sigma_{i}, T_{i}\right)}
$$

where, $x_{i}$ : the time spent by the component at loading parameters $\left(\sigma_{i}, T_{i}\right)$.

The dispersion of the equivalent loading parameter can be approximated by formula

$$
\begin{aligned}
V\left[\mathcal{T}_{e f}\right]= & {\left[c\left(\log \frac{1}{\sum_{(i)} \xi_{i}}-a\right)^{-1} \frac{1}{2.3 \sigma_{i}}\right]^{2} V[\sigma] } \\
& +\left[\left(b+c \log \sigma_{i}\right)\left(\log \frac{1}{\sum_{(i)} \xi_{i}}-c\right)^{2}\right. \\
& \left.\times \frac{\sum_{(i)} \xi_{i}}{2.3 /\left(\sum_{(i)} \xi_{i}\right)}\right]^{2} V\left[\sum_{(i)} \xi_{i}\right]
\end{aligned}
$$

assuming the dispersions of the individual stochastically independent quantities do not exceed $10 \%$ (Ref. 5)).

The contribution of the stress state $V[\sigma]$ can be either evaluated numerically by a stochastic modification of the stress analysis procedure (see, e.g., Ref. 6)) or estimated by a fixed coefficient of variation in the first approximation. The dispersion of the cumulative operational times $V\left[\Sigma_{(i)} \xi_{i}\right]$ is assessed by combining (Ref. 5)) the partial dispersions of the materials regression Eqs. (6) and those of the oper- 
ational times indicated in the neighbourhood of parameters $\left(\sigma_{i}, T_{i}\right)$ (following from inaccuracies of the measuring equipment).

\subsection{A Computerized Operational Data Acquisition and Processing System for Power Units Steamlines}

Piping systems of steam lines of power generating plants are of major importance for operational safety and reliability of both conventional and nuclear installations. In view of their significance in this respect, regular re-assessments are performed in Czechoslovakia of the residual service life of these systems of each individual unit in operation. The mathematical model used for these combines the FEM approach with procedures capable to account for stress relaxation and time-dependent low-cycle fatigue processes to obtain the quasi-static response of the piping system.

This procedure is capable to account also for stochastic fluctuations of the relevant input parameters treated as Gaussian variables. The residual service life is determined as a random quantity the parameters of which result from evaluation of the limit condition of allowable failure probability in the most seriously loaded location of each system. This probability is assessed by quantification of the overlap of the time-dependent random quantities of operational loading and load-bearing capacity in each particular location. For the two most important materials degradation mechanism in the present application, i.e., low-cycle fatigue and creep, stochastic modification of regression curves of integral materials parameters was used (scatter bands of the type of Eq. (6)).

Besides parameters defining the pipeline structurally and geometrically, characteristics of the history of service loading represent the essential inputs of the life-time assessment methodology.

Since the above-defined effective parameter of operational loading is difficult to obtain by conventional methods for routinely operated units, a method of automatic appraisal of the parameter has been proposed. The methodology has been verified by implementation as a permanent section of the monitoring software of a $500 \mathrm{MW}$ coal-firing unit manufactured by Skoda.

The basic working unit of the software package for evaluation of the parameter of effective temperature (as the one representative of the operational loading history in the region of quasi-static creep) is represented by a three-dimensional array storing the acquired operational information of each subsystem. The operational data are stored in the form of relative frequency histograms, such that each element of the array, $H(i, j, k)$ represents the cumulative time interval spent by the subsystem in the $k$-th measured point in a predefined vicinity of the nominal temperature and internal pressure $\left(T_{i}, p_{j}\right)$. Elements of the last column of the array $H(i, j, k)$ serve for recording the number of transitions indicated in the $k$-th measuring location between the $i$-th and $(i+1)$-th temperature bands, i.e., for storage of information necessary for implementation of low-cycle fatigue package. The flow-diagram of the algorithm is presented in Fig. 2.
By these means, residual service-times at $1 \%$ probability of failure by creep rupture of the monitored steam lines have been computed. Thus both the utility (the Gzech Electricity Generating Board) and the manufacturer (Skoda Pilsen) have been provided with a method for routine in-service monitoring of the available service-lives of major components of the pressure boundary based on assessment of the actual service histories of individual systems.

\section{Analysis of Greep Damage by the FE Method}

The above presented non-linear and stochastic problem has been solved also in the case of creep damage of rotors and casings of steam turbines. Step by step linearization of the numerical process in each element of the structure and each time interval has been effected by assuming the linear hypothesis of cumulative damage on the premise of the known real operational temperature and mechanical loading.

For numerical modelling of creep of non-proportionally loaded physical bodies, the theory of internal state variables has been employed. Transition between two creep curves of parameters $\left(\sigma_{1}, \mathcal{T}_{1}\right),\left(\sigma_{2}\right.$, $T_{2}$ ) of the type (1) has been assumed as governed by the level of damage, $D$, incurred by the former of the two process, i.e., that characterized by parameters $\left(\sigma_{1}\right.$, $T_{1}$ ) (Ref. 7)). This principle has been verified by both experiments and practical observations, and is illustrated in Fig. 3.

The finite element method (FEM) with triangular elements (plane stress/strain, rotationally symmetrical and spatial problems) with linear shape functions has been used for establishment of non-stationary temperature fields and of the creep process. The fundamental equation for modelling creep has the form

$$
\{K\}\{\delta\}=\{R\}+\left\{F_{c}\right\}
$$

where $F_{c}$ is additional fictional force which will increase in each time interval $\Delta t$ by $\Delta F_{c}$

$$
\left\{\Delta F_{c}\right\}=[\alpha]^{T} \int_{(v)}[\beta]^{T}\left[D_{c}\right]\left\{d \varepsilon_{c}\right\} d V
$$

In the above equations,

$[K]$ : the overall stiffness matrix

$\{\delta\},\{R\}$ : the column vectors of displacements and external forces, respectively

$\left\{d \varepsilon_{c}\right\}$ : the column vector of creep increments of plastic deformation following from PrandtlReuss relastionships.

Furthermore,

$V:$ the volume of an element

$[\alpha]$ : a matrix constituted by coefficient of the linear polynominal for approximation of displacements within an element

$[\beta]$ : the matrix of differential operators representing the relationships between displacements and components of deformation

$\left[D_{c}\right]$ : the matrix of elasticity

the exponent $T$ : the transposition of a matrix.

The calculation of time intervals, which is the most important factor of any numerical procedure of creep 


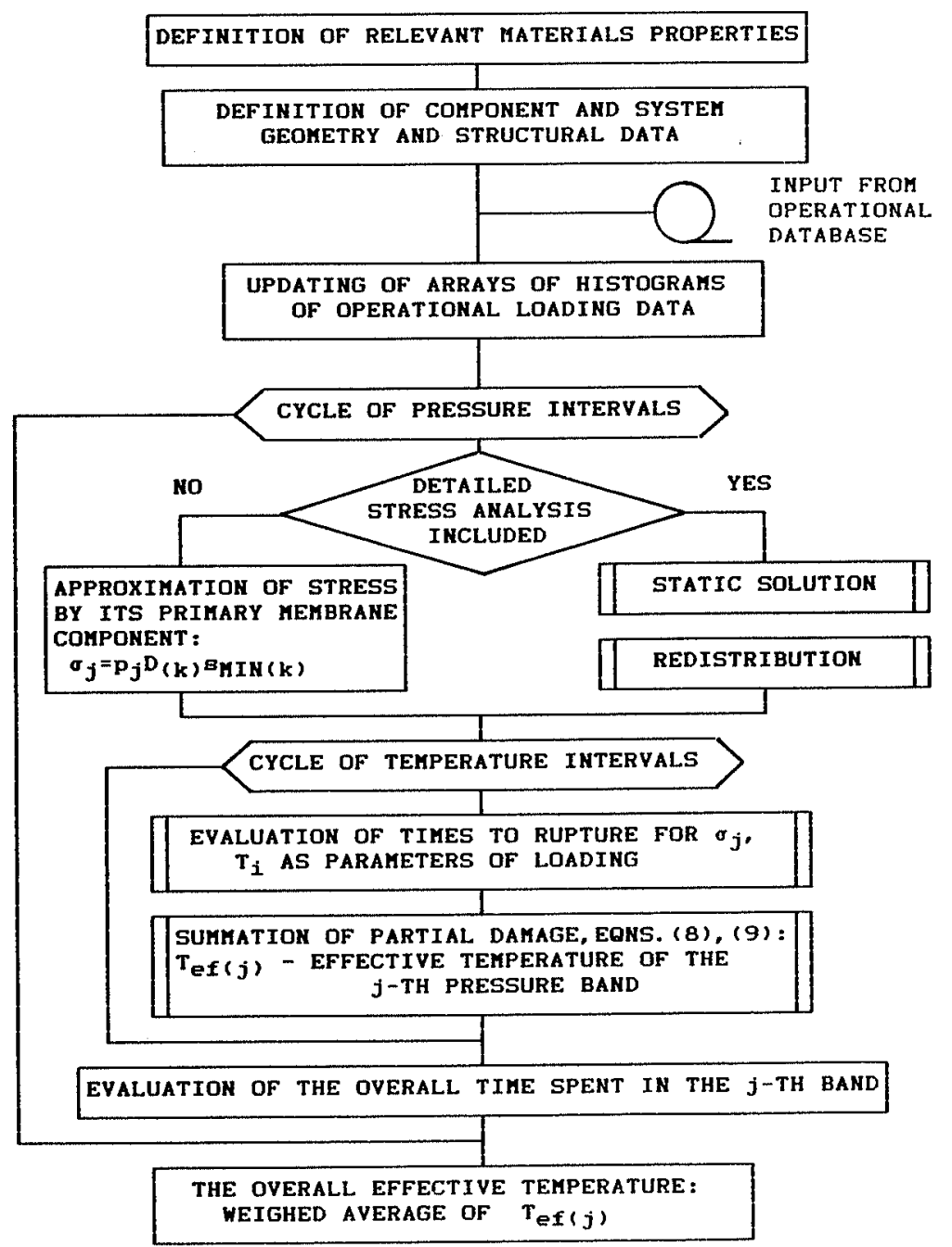

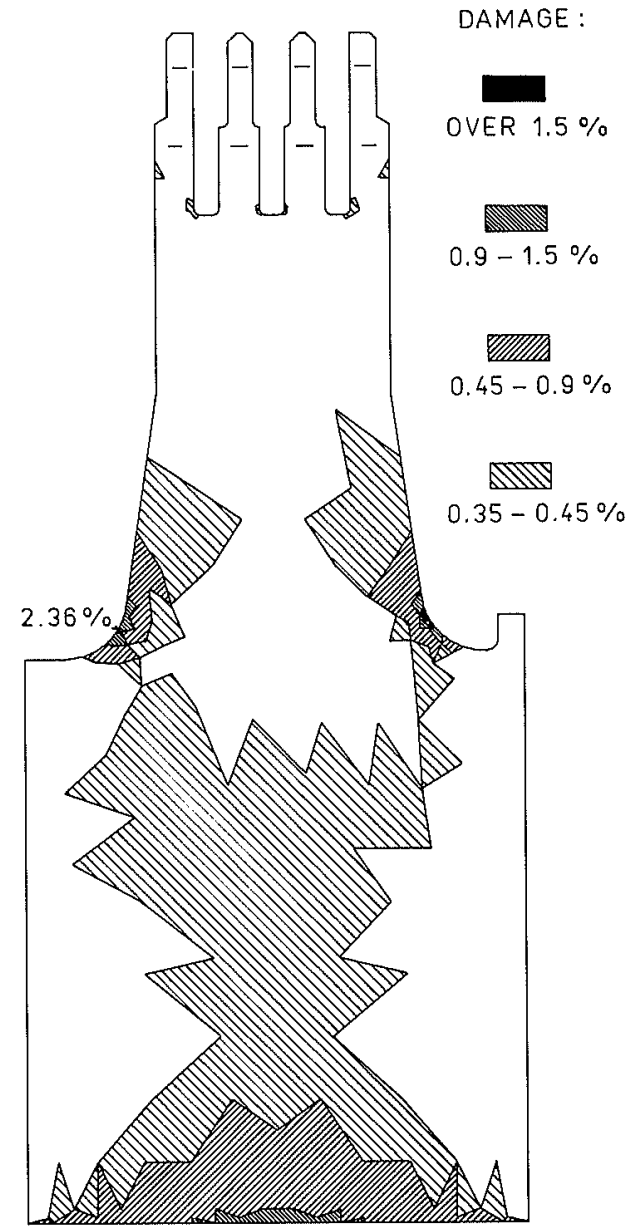

Fig. 4. The pattern of damage in the disc of the control stage of the $200 \mathrm{MW}$ high-pressure rotor after $1.2 \times 10^{5} \mathrm{~h}$ of creep.

Fig. 2. Flow diagram of the procedure for evaluation of the parameter of equivalent loading.

Fig. 3 .

The transition between individual levels of creep curves according to the achieved degree of creep damage.

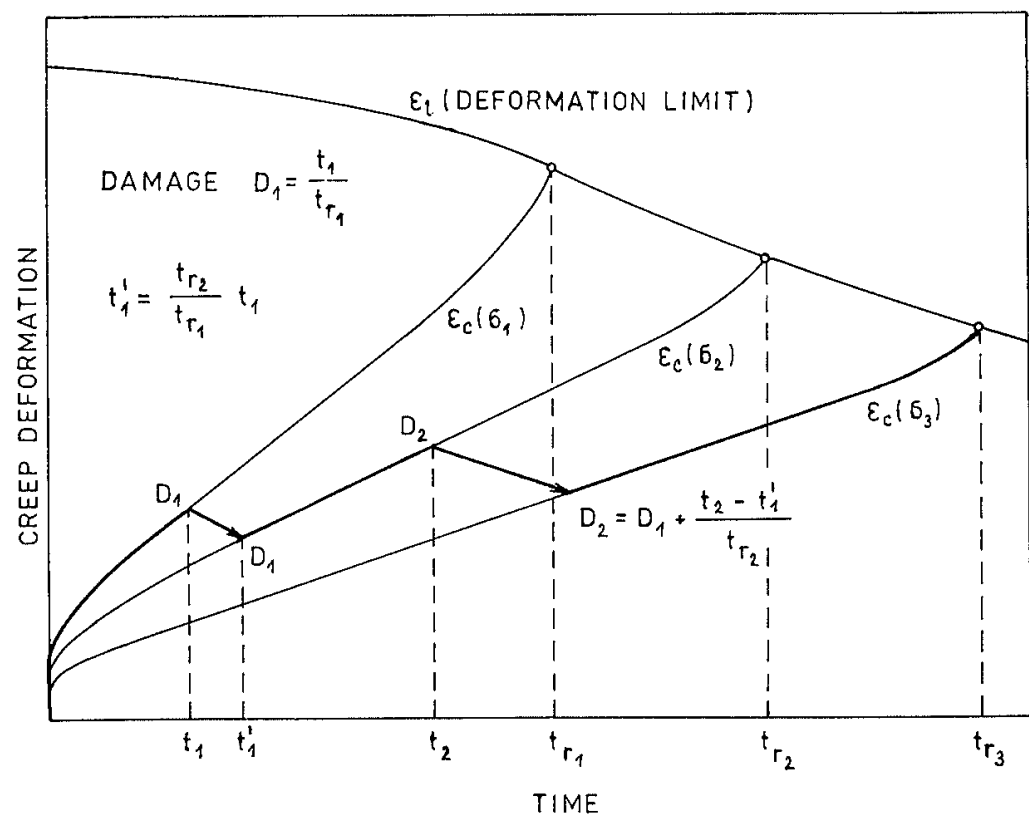

evaluation, is performed automatically by the present programme according to the maximum value of the intensity of stress. From 3 to 5 recurrent solutions are sufficient in each time interval. This method has also been used for assessment of the residual service- life of the first medium-pressure rotor disc of 110 and $200 \mathrm{MW}$ steam turbines manufactured by Skoda and for estimation of the damage by creep of the control stage disc of the $200 \mathrm{MW}$ high-pressure rotor during stationary operation (Fig..4) - The maximum tem- 
perature of $520^{\circ} \mathrm{C}$ is in the region of the clevis root of the blade, while that of $500^{\circ} \mathrm{G}$ is in the boring. For this purpose, a grid of 1072 elements was used. The highest local damage after $1.2 \times 10^{5} \mathrm{~h}$ of operation is located in the left transitional area of the disc into the rotor, with calculated maximum of approximately $2.4 \%$. The corresponding magnitude at the internal surface of the boring is approximately $1 \%$. Fig. 5 shows the re-distribution of stress intensity (in $\mathrm{MPa}$ ) in the first $200 \mathrm{MW}$ medium-pressure disc (1 379 elements used). Fig. 6 shows detailed isostresses at the grooved end of this disc after $4 \times 10^{4} \mathrm{~h}$. As seen from the figure, even after such a relatively long period of operation significant stress concentration occurs in the vicinity of the $\mathrm{T}$-groove (temperature $532^{\circ} \mathrm{C}$ ).

The maximum creep rate at the limit of the scatter band of creep curves was assumed for the material near the rotor boring characterized by worsened creep properties. The model was modified using a correction coefficient of 1.25 for the effective value of stress in relationships (1) to (4).

The computed results of plastic deformation of the rotor boring under the first disc have been found to be in agreement with two measurements having been performed for two steam turbine rotors of the above mentioned types under stationary operational conditions. The plastic deformation of the $110 \mathrm{MW}$ rotor boring after $1.12 \times 10^{5} \mathrm{~h}$ of creep obtained by the numerical procedure was $0.037 \%$. The amount of plastic deformation measured in power plant in Ludus (Romania) after $1.12 \times 10^{5} \mathrm{~h}$ of operation in this location is about $0.04 \%$.

In another application of this numerical process, the procedure was used to assess creep damage of the internal high-pressure $200 \mathrm{MW}$ steam turbine casing following cold start-up (interruption of operation for

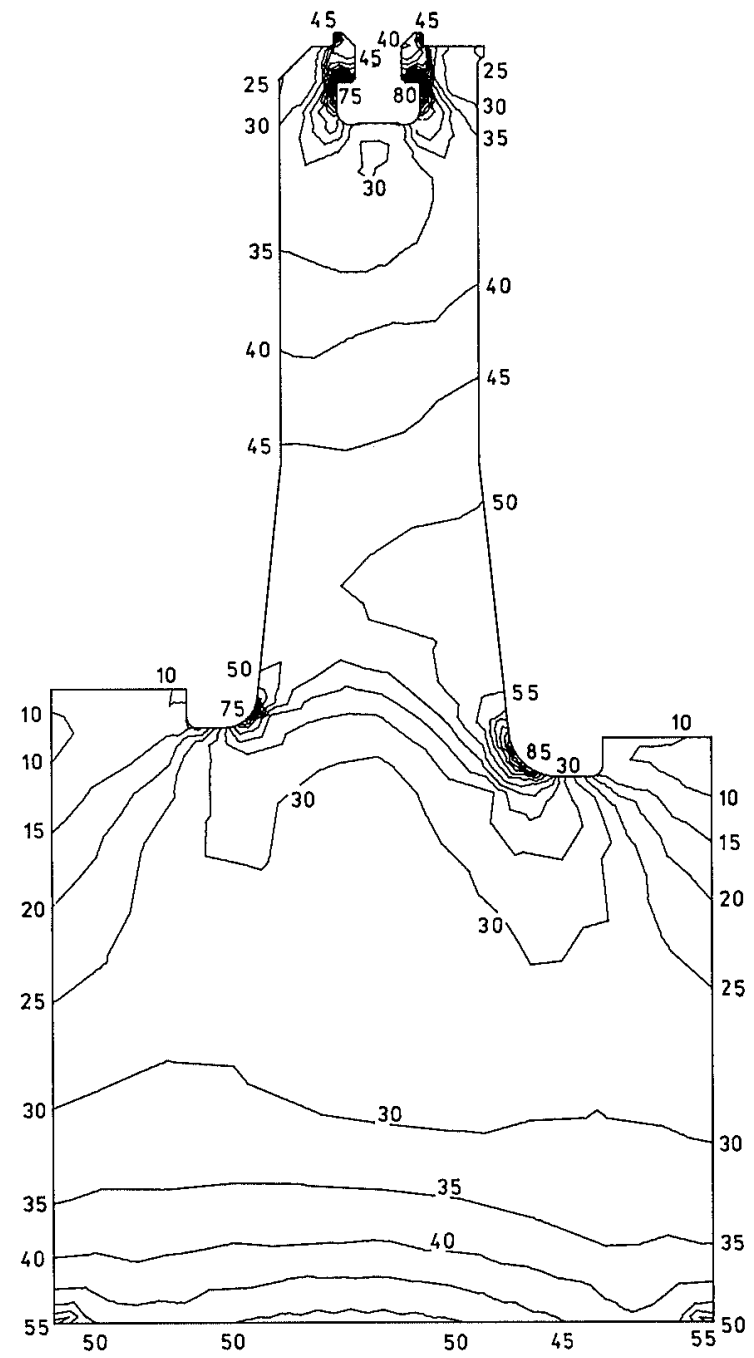

Fig. 5. The shape of isolines of the stress intensity in the first $200 \mathrm{MW}$ medium-pressure disc after $4 \times 10^{4} \mathrm{~h}$ of creep.

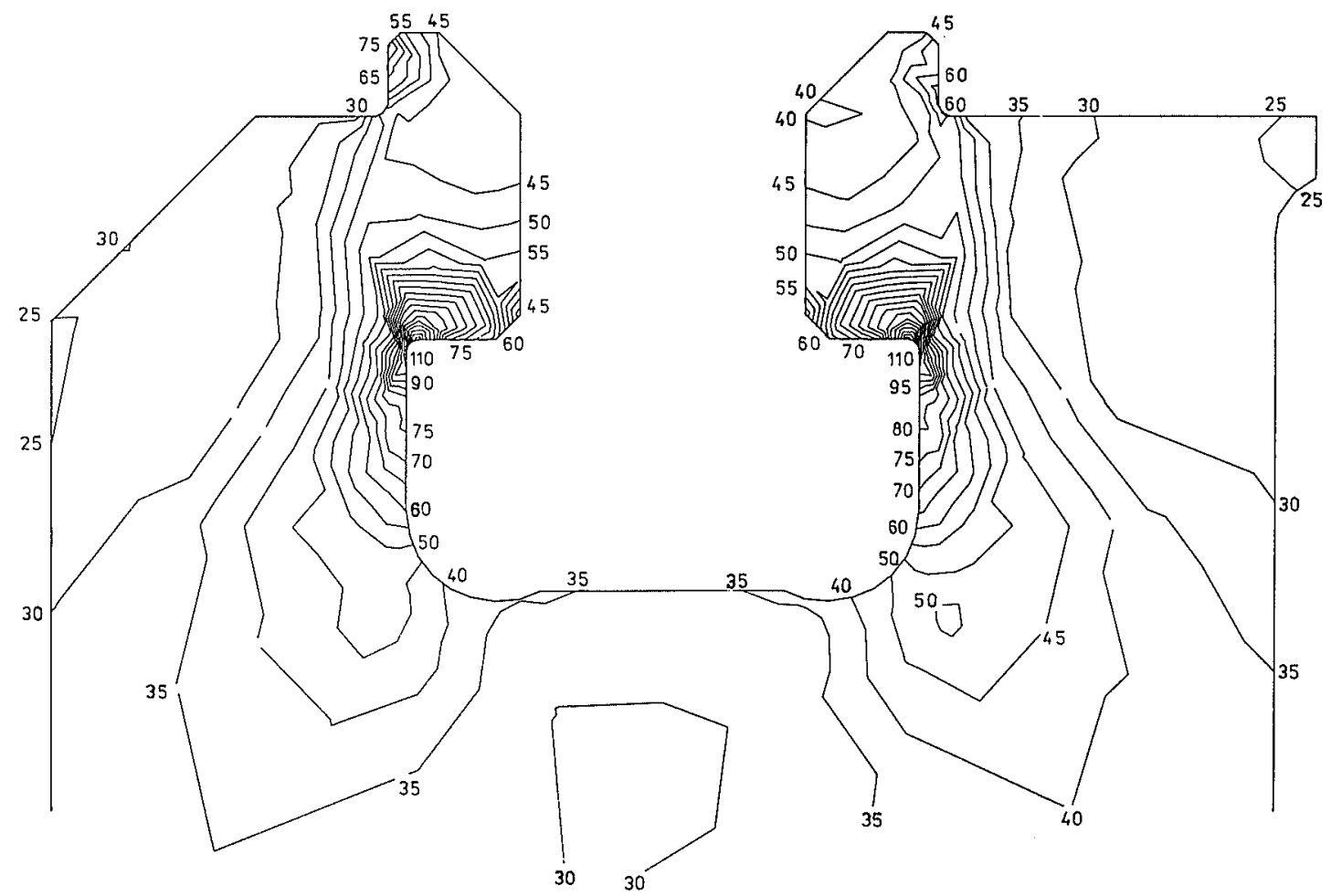

Fig. 6. The detailed isolines of the stress intensity at the grooved end of disc after $4 \times 10^{4} \mathrm{~h}$ of creep. 


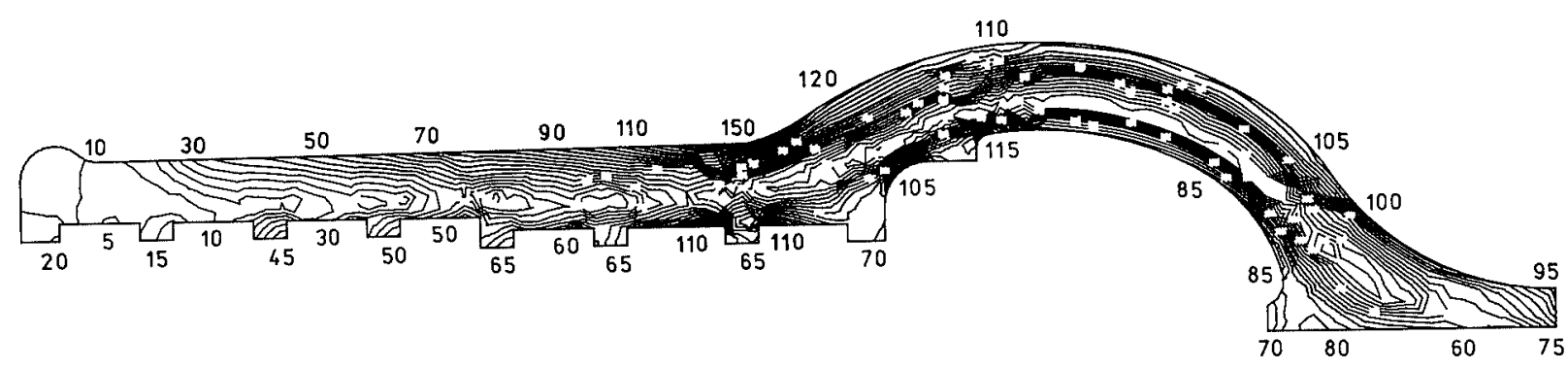

Fig. 7. The shape of isolines of the stress intensity in the internal $200 \mathrm{MW}$ high-pressure casing after $10^{4} \mathrm{~h}$ of creep.

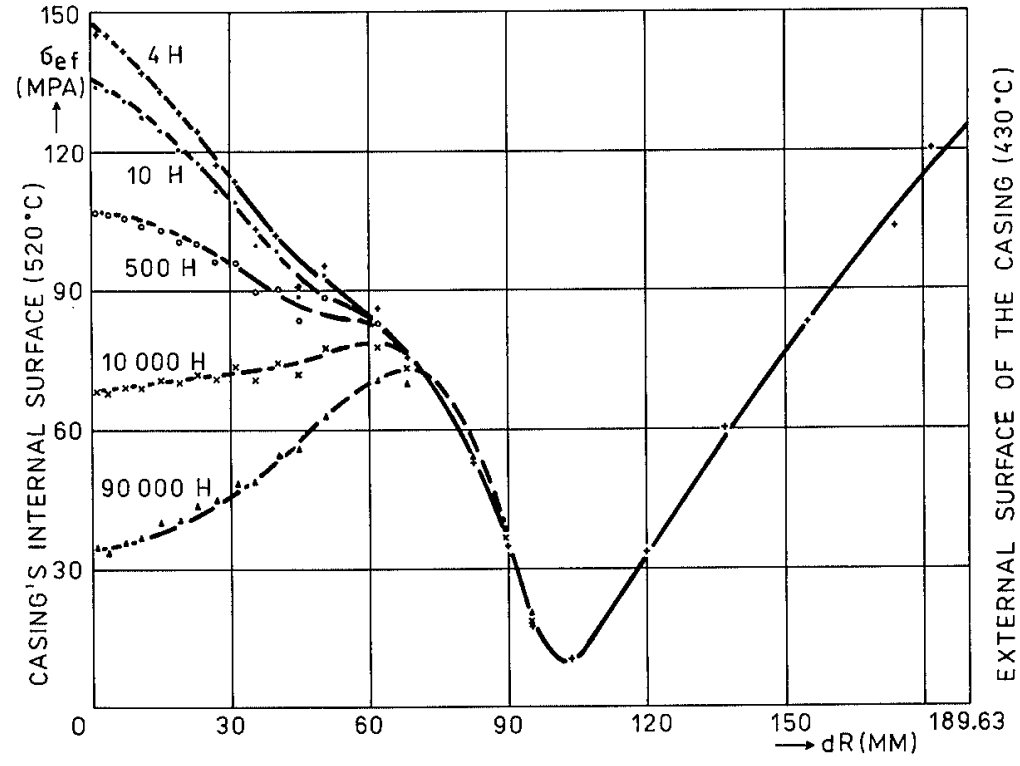

Fig. 8.

The redistribution of stress in a cross-section perpendicular to the axis of symmetry in front of the first impeller wheel. more then $48 \mathrm{~h}$ ) under non-proportional conditions of loading ( $4 \mathrm{~h}$ starting-up interval from steam temperature $180^{\circ} \mathrm{C}$ with the corresponding starting rate of approximately $85^{\circ} \mathrm{C}$ per hour). A rotationally symmetrical model was used to model the casing. The creep limit temperature of material was approximately $470^{\circ} \mathrm{C}$. Fig. 7 shows the isolines of the stress intensity (according to the Huber-von Mises-Hencky hypothesis) in $\mathrm{MPa}$ after $10^{4} \mathrm{~h}$ of operation following the start-up procedure. Large decrements of the stress gradients can be observed in the corners of projections in front of the first partition and at the opposite side of the spherical section of the casing. High density of isolines can be observed in the vicinity of the rounded grooves of the casing cap and on the left side, in the location of the minimum wall thickness. Increased stress is also visible at the external surface of the casing in the region of shape transition between the linear and circular sections. Fig. 8 shows the changes of the stress intensity during creep in a cross-section of the casing in front of the partition.

\section{Conclusions}

A detailed analysis of rotors and casings of steam turbines in creep regime was performed based on extensive theoretical and experimental studies using ad- vanced computer programmes. Levels of material damage by creep and plastic deformations have been assessed in critical locations of the components. The numerical results have been used for assessment of the actual service lives of components available from the view-point of future reliable operation.

\section{REFERENCES}

1) A. F. Armor, R. J. Jaffee and G. L. Touchton: "Improved Coal-fired Power Plants-A New Technology Thrust for U.S. Utilities", Am. Power. Conf., Chicago, 1986.

2) Ist Int. Conf. on Improved Coal-fired Power Plants, EPRI, Palo Alto, (1986).

3) V. Bina and R. Pech: Machinery Eng. (Strojirenstvi), 30 (1980), 227, (in Czech).

4) R. Ohtani and T. Kitamura: Tetsu-to-Hagané, 72 (1986), 711.

5) E. B. Haugen: Probabilistic Approaches to Design, John Wiley \& Sons, New York, (1982), 130-156.

6) M. Svarc: "An Operational Data Acquisition System for Service-life Assessment of Piping Components", IX Conf. Energiewirtschaft, May 1989, Technische Hochschule Zittau, DDR, Paper No. 111-16.

7) J. Valenta and J. Matoušek: "On the Creep Stress Analysis and Life-time of Some Structures", The 1974 Symp. on Mech. Beh. on Materials, August 1974, Kyoto, Soc. of Mater. Sci., Full Manuscripts, Session II-5 (1). 